\title{
ECRH AND W7-X: AN INTRIGUING PAIR
}

\author{
V. Erckmann ${ }^{\mathrm{a}}$, H. Braune ${ }^{\mathrm{a}}$, G. Gantenbein ${ }^{\mathrm{b}}$, J. Jelonnek $^{\mathrm{b}}$, W. Kasparek ${ }^{\mathrm{c}}$, \\ H. P. Laqua ${ }^{\mathrm{a}}$, C. Lechte ${ }^{\mathrm{c}}$, N. B. Marushchenko ${ }^{\mathrm{a}}$, G. Michel ${ }^{\mathrm{a}}$, B. Plaum ${ }^{\mathrm{c}}$, \\ M. Thumm ${ }^{\mathrm{b}}, \mathrm{M}$. Weissgerber ${ }^{\mathrm{a}}, \mathrm{R}$. Wolf ${ }^{\mathrm{a}}$, and the W7-X ECRH- teams \\ at IPP Greifswald ${ }^{\mathrm{a}}, \mathrm{KIT}^{\mathrm{b}}$, and IGVP Stuttgart ${ }^{\mathrm{c}}$
}

\author{
${ }^{a}$ Max-Planck-Institut für Plasmaphysik (IPP), EURATOM-Association, D-17491 Greifswald, Germany \\ ${ }^{b}$ Karlsruher Institut für Technologie, KIT, Association EURATOM-KIT, IHM, D-76021 Karlsruhe, Germany \\ ${ }^{c}$ Institut für Grenzflächenverfahrenstechnik und Plasmatechnologie, (IGVP) Universität Stuttgart,
} D-70569 Stuttgart, Germany

\begin{abstract}
The construction of the W7-X basic machine is almost completed and the device is approaching the commissioning phase. W7-X operation will be supported by ECRH working at $140 \mathrm{GHz}$ in $2^{\text {nd }}$ harmonic X- or O-mode with $10 \mathrm{MW} \mathrm{cw}$ power. Presently the activities at W7-X concentrate on the implementation of wall-armour, in-vessel components and diagnostics. The ECRH-system is in stand by with 5 out of 10 gyrotrons operational. The status of both, the W7-X device and the ECRH system is reported. Further R\&D activities concentrate on extending the launching capability for sophisticated confinement investigations with remote steering launchers in a poloidal plane with weak magnetic field gradient.
\end{abstract}

Keywords: ECRH, Stellarator, Microwaves

PACS: 52.50 .-b $84.40 . \mathrm{Ik} 52.35 . \mathrm{Hr}$

\section{INTRODUCTION}

Wendelstein 7-X $(\mathrm{R}=5.5 \mathrm{~m},<\mathrm{a}>=0.55 \mathrm{~m})$ aims at demonstrating the reactor capability of the stellarator concept at fusion relevant parameters of $\mathrm{T}_{\mathrm{e}}=2-10 \mathrm{keV}, \mathrm{T}_{\mathrm{i}}=2-5 \mathrm{keV}$ and $\mathrm{n}_{\mathrm{e}}=0.1-310^{20} \mathrm{~m}^{-3}$, and a plasma $\beta$ of about $5 \%$ $[1,2]$. Steady state operation is an intrinsic capability of stellarators. A set of superconducting (SC) coils generates the 3-D magnetic configuration of W7-X, which is optimized for good confinement, robust equilibria and high- $\beta$ stability. The nested magnetic surfaces of the central confinement region are separated from a chain of natural islands, which constitute the divertor configuration at the plasma boundary. Pfirsch-Schlüter and bootstrap currents are minimized, resulting in a robust equilibrium with weak dependence on plasma- $\beta$. The nominal magnetic field is $2.5 \mathrm{~T}$, the magnetic configuration has 5 field periods, operates around $\iota=1$ and is generated by a modular coil system consisting of 50 non-planar coils. Experimental flexibility is provided by the possibility to power the 5 groups of non-planar coils with different current, thus generating configurations with different magnetic mirror ratio. An additional set of 20 planar coils serves for tuning the rotational transform and generates a small vertical field for plasma position shift. A set of 5 normal conducting external correction coils allows fine tuning of the divertor X-points and compensation of field errors, if necessary. The W7-X divertor is designed for the removal of a heating power of $10 \mathrm{MW}$ for 30 minutes plasma operation. ECRH is the main heating system for cw-operation, the power is generated by ten $140 \mathrm{GHz}$ gyrotrons with about $1 \mathrm{MW}$ of microwave power each. In addition, neutral beam injection and ion cyclotron heating are foreseen to probe the beta and equilibrium limits in pulsed $10 \mathrm{~s}$ operation and to study fast ion confinement and fast ion driven instabilities.

\section{W7-X ASSEMBLY STATUS}

The W7-X cryostat consisting of the plasma vessel and the outer vacuum chamber with the embedded SC-coil system is completed with all current feed-throughs and bus-bars functional. All ports are installed. Fig.1 shows the CAD design (left) and a section of the completed device in the torus hall (right).

The subsystems such as the cryogenic plant, the central water cooling system, the DC-power supplies for the SCcoils, the quench detection and emergency discharge systems are completed and in stand-by for the commissioning phase. The activities now concentrate on the completion of the in-vessel installation, i.e. first wall armour, the 
cooling and support structure for the later step-by-step implementation of the test divertor unit and, later on, the actively pumped high heat load divertor with full steady state capability. The diagnostic system for the vacuum magnetic flux-surface measurements and a selected set of plasma diagnostics for the first operation phase are being installed together with protective diagnostics to guarantee machine operation safety. The first operation phase is planned without divertor to allow for flux surface measurements without aperture limiting plasma-facing components. The divertor structure and the baffles would cut into the magnetic islands at the plasma edge and thus limit the desired information about position, size, shape, and symmetry of the magnetic island structure for the different magnetic configurations. The flux surface measurements will be followed by a low-performance plasma operation to check the proper functioning and interplay of all required subsystems. The plasma will then be defined by a limiter type edge, depending on the chosen configuration. The absence of a divertor in the first operation phase limits the plasma performance to a maximum energy input of about 2 MJ. Plasma generation and heating will thus be provided by ECRH with moderate power and pulse length.
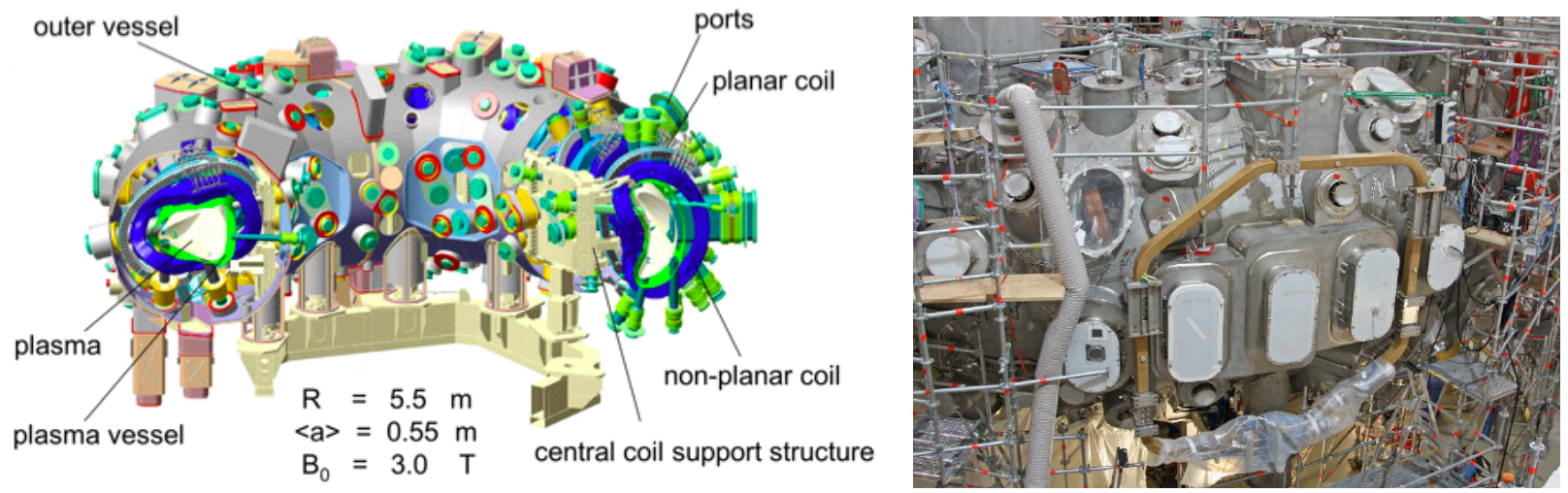

FIGURE 1. Left: Principle sketch of W7-X showing the support structure, the inner and outer vacuum vessel, the SC-coils and the plasma cross-section. Right: Section of the completed device in the torus hall. One normal-conducting correction coils is seen surrounding the triplet of large ports. Two of them are used in two modules for ECRH equatorial launch.

The most exposed wall sections will be provisionally shielded by properly arranged discrete elements without active cooling. The first commissioning phase, will then be followed by a scheduled shutdown for the installation of the inertially cooled test divertor unit and baffle assembly. Maximum advantage can thus be taken from the flux surface measurements and the first plasma operation to optimize the divertor position and the control system for $\mathrm{X}$-point control. The commissioning phase of the supporting components is scheduled for mid 2014 with the aim to evacuate and leak-check both the plasma vessel and cryo-vacuum. First operation of the cryo-plant and subsequent cool-down of the W7-X cold mass (coil support structure and coils) towards L-He temperature and charging of the coils with nominal current are the next steps. A reduced version of the central control system was implemented in the WEGA-Stellarator experiment and pilot tests were performed successfully. A downgraded version of the CoDaC-system will be implemented for controlled W7-X operation in the commissioning phase. Magnetic flux surface measurements will be performed with different methods [3] with the magnets charged to nominal field. Wall conditioning using glow discharge cleaning (no magnetic field) and rf-methods [4] will follow. First integrated plasma operation will be tested directly after completion of the flux-surface measurement and removal of the related diagnostics equipment. This operation phase aims at demonstrating the proper functioning and interplay of all major machine components including ECRH and a first set of diagnostics. The subsequent shut-down will see the implementation of the test divertor unit, an improved set of diagnostics, further improvement and debugging of the central $\mathrm{CoDaC}$-system towards real-time processing performance.

\section{STATUS OF ECRH FOR W7-X}

ECRH is the main heating system for steady state operation. It provides a large flexibility and complies with various physics demands such as controlled plasma start-up from the neutral gas, steady state plasma control, and performance optimization by plasma profile shaping. The narrowly localized power deposition, the technological advantage of remote wave launching and beam control, and, last not least, the good theoretical understanding of the wave-particle interaction physics assigns a key role to ECRH, which will support W7-X operation with $10 \mathrm{MW}$ 
heating power at $140 \mathrm{GHz}[5]$. The standard heating and current drive scenario is X2- mode $\left(\mathrm{B}_{\text {res }}=2.5 \mathrm{~T}\right)$ with low field side launch (LFS). High-density operation above the X2 cut-off density of $1.2 \times 10^{20} \mathrm{~m}^{-3}$ is accessible with O2-mode $\left(<2.5 \times 10^{20} \mathrm{~m}^{-3}\right)$ and at even higher densities with O-X-B mode conversion heating [6], respectively. $\mathrm{X}$ 3-mode heating $\left(\mathrm{B}_{\mathrm{res}}=1.66 \mathrm{~T}, \mathrm{n}_{\mathrm{e}}<1.6 \times 10^{20} \mathrm{~m}^{-3}\right)$ is a promising scenario for operation at reduced magnetic field, which extends the operation-flexibility. Reliable plasma start-up with X3-mode has, however, not been demonstrated yet. As W7-X has no OH-transformer for inductive current drive, EC-current drive is a valuable tool to modify the internal current density distribution and to counteract residual bootstrap currents. The main components of the entire system such as cooling system, SC-magnets with the related PS and cryo-system, HVmodulators for the output-power control and fast modulation are tested at full performance and are ready for operation. The rf-power is transmitted from the gyrotrons to W7-X via quasi-optical mirror based transmission systems with cw-capability and low loss. One reference line was tested over the full length $(50-60 \mathrm{~m})$ at $0.7 \mathrm{MW}$ power in cw-operation showing losses of $2.6 \%$, which is close to the theoretical limits. The front end of the transmission line can, however, only be tested at high power after installation in the torus ports during the commissioning phase of W7-X. One out of four plug-in launcher units is seen from Fig. 2 (left), which fits into the large ports as seen from Fig. 1 (right). All launchers are leak tested and ready for installation. Two ECRH-towers house the transmission line components, which are located near the torus in the experimental hall, in particular the beam distribution optics as well as the launcher control and data acquisition module. The towers are built as "plug and play' units and are ready for transportation to and installation in the torus hall.
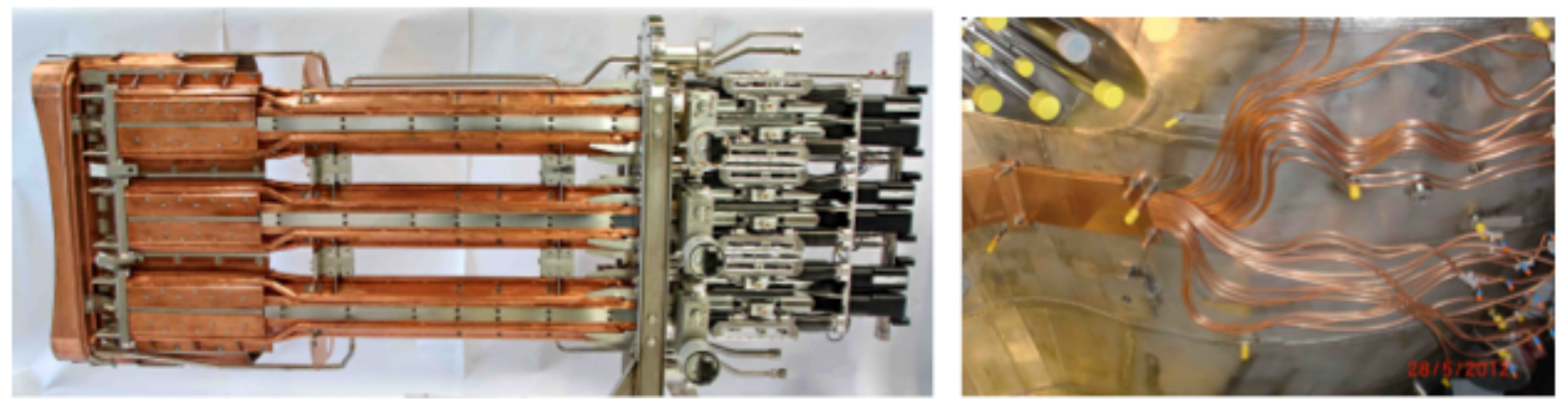

FIGURE 2. ECRH related in-vessel structures. Left: Front steering plug-in launcher transmitting 3 microwave beams with $1 \mathrm{MW}$ each. The actively cooled beam shielding structure for cw-operation is clearly seen. Right: One out of four ECAwaveguide bundles installed at the inner torus wall opposite to the plug in launchers.

A point of concern is still the availability of the gyrotrons, which are provided by THALES ( 9 units) and CPI ( 1 unit). Up to now only 5 out of altogether 10 gyrotrons are installed and ready for operation. The successful development of prototypes was followed by unexpected difficulties reproducing the prototype achievements in series production [7]. The delivery of additional two THALES series gyrotrons is expected until the start of the commissioning phase, followed by the CPI gyrotron and one more THALES tube. Maximum benefit will be taken for the EU-development of the $170 \mathrm{GHz}$ ITER gyrotron from the painful process of recovering the prototype achievements in series production for W7-X. The design is derived from the W7-X design and major improvements such as the corrugated beam tunnel, radial collector sweep and reflective coating of inner structures are identical or close to the proven technology implemented in the W7-X gyrotron. Major difference is the higher-order operation mode $\mathrm{TE}_{32,9}\left(\mathrm{TE}_{28,8}\right.$ for the $\mathrm{W} 7-\mathrm{X}$ gyrotron), and an improved efficiency to meet the ITER requirement of $>50 \%$.

\section{REMOTE STEERING LAUNCHER FOR ADVANCED CONFINEMENT STUDIES}

In both, the W7-X and ITER devices, the ECRH power is launched through quasi-optical front-steering launchers. These launchers provide a wide angular steering range and a narrow focusing of the individual RF beams. In ITER the wide steering range is required for MHD stabilization at $\mathrm{q}=1,1.5,2$, whereas for $\mathrm{W} 7-\mathrm{X}$ it is mandatory for the high-density H\&CD scenarios with O-X-B mode conversion heating. Advanced confinement investigations probing the velocity space are possible at W7-X by comparing standard launch with wave absorption in a poloidal plane with almost vanishing magnetic field gradient. The physics of wave particle interaction in this plane is very sensitive to trapped particles. Note, that $|\mathrm{B}|$ varies strongly along the magnetic axis in $\mathrm{W} 7-\mathrm{X}$ within one field period. 
An example for ray tracing calculations with $140 \mathrm{GHz}, \mathrm{X} 3$-mode heating at $\mathrm{B}_{\text {res }}=1.67 \mathrm{~T}$ is seen from Fig. 3 (right) for two launch angles with strong absorption by either passing $\left(+15^{\circ}\right)$ or trapped particles $\left(0^{\circ}\right.$, i.e. wave propagates along the waveguide axis). The target plasma is generated with $4 \mathrm{MW}$ X2 heating at $104 \mathrm{GHz}$ in the bean-shaped plane, where the corresponding magnetic field is $1.85 \mathrm{~T}$. The 'zero-gradient' plane is accessible only through narrow $\mathrm{N}$-ports, where front steering launchers as used in the A and E ports (see Fig. 1 (right)) would not fit. An alternative concept with attractive features is the 'remote steering launcher (RSL)', which is based on multi-mode interference in a square waveguide leading to imaging effects: For a proper length of the waveguide, a microwave beam at the input of the waveguide (with a defined direction set by a mirror system outside of the plasma vacuum) will exit the waveguide (near the plasma) in the same direction. The obvious advantage of this approach is that the beam steering mechanism can be placed outside the plasma chamber and the plasma facing in-vessel structure is a rigid waveguide with no moving parts. This is a desirable and robust solution in view of future applications in fusion devices with burning plasmas like DEMO. Furthermore, the power density (port through power/ port area) for RSLs would be typically $100 \mathrm{MWm}^{-2}$ as compared to $8-11 \mathrm{MWm}^{-2}$ of the front-steering systems at W7-X and ITER. A principle sketch of the arrangement is seen from Fig. 3 (left). Motivated by both, physics and technology reasons, two out of the 10 microwave beams at W7-X can be switched to RSLs.
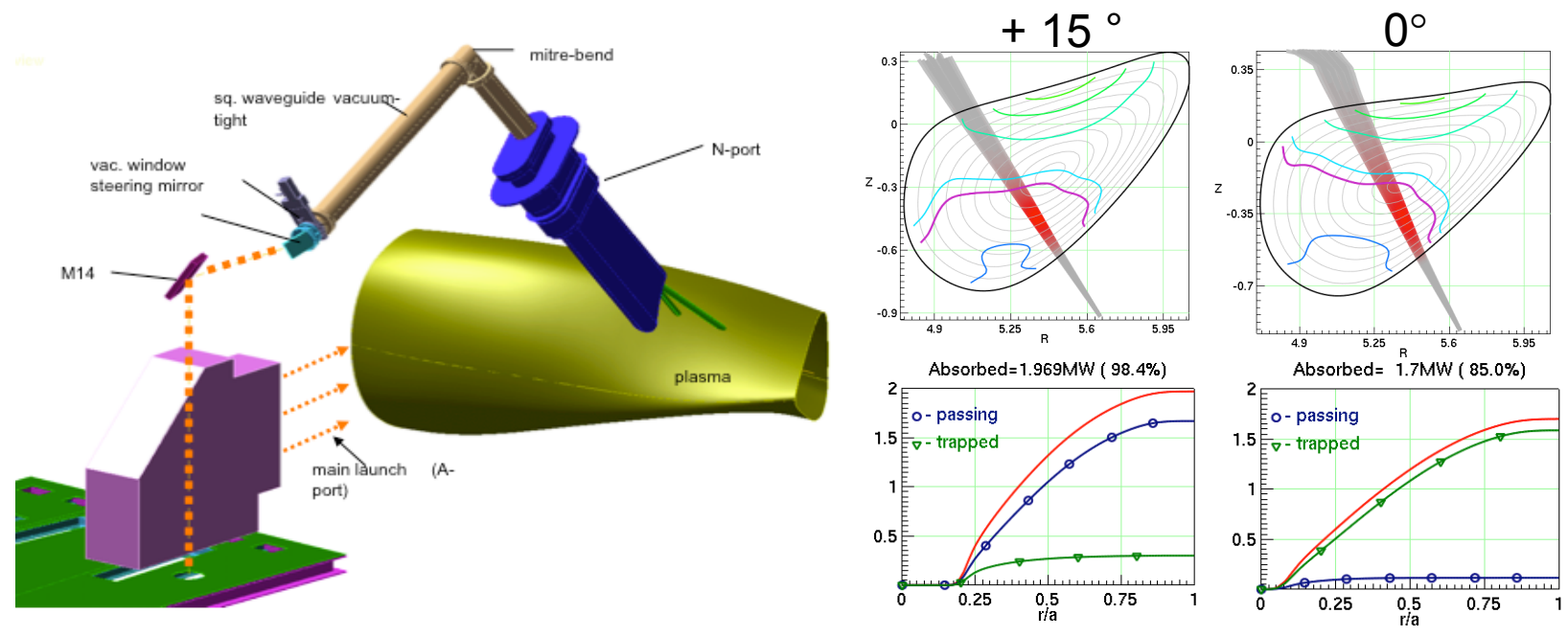

FIGURE 3. Left: Remote steering launcher transmitting one microwave beam towards a poloidal plane (N-port) with small magnetic field gradient. Right top: Ray tracing of the launched beam for two launch angles in this plane.

Right bottom: Absorbed power from trapped and passing electrons and total absorbed power for both launch angles.

The RSLs will be fed from the main transmission as sketched in Fig.3 (left). As the imaging characteristics of square waveguides diminish at steering angles $>12^{\circ}$, we have started an R\&D-programme to optimize RSLs with respect to steering range and beam quality [8,9]. One RSL will employ a square corrugated waveguide with an optimized control of the beam injection at the waveguide entrance. For the other RSL, a waveguide with a modified cross-section is foreseen. For the optimization, a new simulation code was developed, which allows the simultaneous calculation of all propagation constants in the deformed waveguide; calculations have started to find the best-performing RSL waveguide cross-section. The waveguides will be fabricated by electroforming with embedded cooling channels; commissioning of the RSLs is planned for 2015.

\section{REFERENCES}

1. C.D. Beidler, et.al., Fusion Techn. 17 (1990) 148

2. R.C. Wolf, et al., Fusion Eng, Design 83 (2008) 990

3. Otte et al., private communication

4. T. Wauters, et al., this conference

5. V. Erckmann et al., Fusion Science and Technology, Vol. 52, No.2 (2007) pp. 291-312

6. H. P. Laqua, V. Erckmann, H.J. Hartfuß, H. Laqua, W7-AS Team, and ECRH Group: Phys. Rev. Lett. 78, 3467 (1997)

7. J. Jelonnek, et al., Proc. $37^{\text {th }}$ IRMMW-THz 2012, Australia, 2012

8. K. Ohkubo et al., Fusion Eng. Design 65 (2003) 657-672

9. G.G. Denisov, K.A. Fedorova, et al., Int. J. Infrared Milli Waves (2007) 28:923-935 